\title{
EXPEDIENTE
}

\section{EDITOR CIENTÍFICO}

Prof. Dr. Álvaro da Silva Santos

\section{COMITÊ DIRETOR}

Profa. Dra. Grasielle Silveira Tavares Paulin Profa. Dra. Marta Regina Farinelli

Profa. Dra. Renata Damião

\section{COMITÊ EDITORIAL}

Prof. Dr. Acir Mário Karwoski - UFTM/MG

Prof. Dr. Álvaro da Silva Santos - UFTM/MG

Profa. Dra. Ana Lúcia de Assis Simões - UFTM/MG

Prof. Dr. Gilberto de Araújo Pereira - UFTM/MG

Profa. Dra. Grasielle Silveira Tavares Paulin - UFTM/MG

Profa. Dra. Jurema Ribeiro Luiz Gonçalves - UFTM/MG

Profa. Dra. Lislei Jorge Patrizzi - UFTM/MG

Profa. Dra. Marta Regina Farinelli - UFTM/MG

Profa. Dra. Renata Damião - UFTM/MG

Profa. Dra. Rosane Aparecida de Sousa Martins - UFTM/MG

Profa. Dra. Rosimar Alves Querino - UFTM/MG

Profa. Dra. Yeda Aparecida de Oliveira Duarte - USP/SP

\section{CONSELHO EDITORIAL}

Prof. Dr. Ailton de Souza Aragão - UFTM/MG

Profa. Dra. Ana Ligia Leandrini de Oliveira - USP/SP

Profa. Dra. Celita Salmaso Trelha - UEL/ PR

Profa. Dra. Claúdia Helena Julião - UFTM/MG

Profa. Dra. Divanice Contim - UFTM/MG

Profa. Dra. Erika Aparecida da Silveira - UFG/GO

Profa. Dra. Estefânia Maria Soares Pereira - UFTM/MG

Prof. Dr. Fábio Almeida - Virginia Tech University/EUA

Prof. Dr. Fábio Scorsolini-Comin - UFTM/MG

Profa. Dra. Geni de Araújo Costa - UFU/MG

Prof. Dr. Hélio Moraes de Souza - UFTM/MG

Profa. Dra. Isabel Aparecida Porcatti de Walsh - UFTM/MG

Prof. Dr. Jair Sindra Virtuoso Júnior - UFTM/MG

Prof. Dr. Jonas Lírio Gurgel - UFF/RJ

Profa. Dra. Julia Elba de Souza Ferreira - UFTM/MG

Profa. Dra. Leiner Resende Rodrigues - UFTM/MG

Prof. Dr. Manuel Antonio Velandia - Universidad de Alicante - Espanha

Profa. Dra. Maria Alice S. Curado - Escola Superior de Enfermagem de Lisboa

Profa. Dra. Maria Del Carmen Solano Ruiz - Universidad de Alicante/Espanha

Profa. Dra. Maria Cecília Leite Moraes - UNASP/SP

Profa. Dra. Maria Lúcia Lebrão - USP/SP

Profa. Dra. Marília Ferreira Dela Coleta - UFU/MG

Prof. Dr. Mário Alfredo Silveira Miranzi - UFTM/MG

Prof. Dr. Mario León Silva-Vergara - UFTM/MG

Prof. Dr. Paulo Fernando de Souza Campos - UNISA/SP

Profa. Dra. Raquel Souzas - UFBA/BA

Profa. Dra. Rosali Isabel Barduchi Ohl - UNIFESP/SP

Profa. Dra. Suely Godoy Agostinho Gimeno - UNIFESP/SP

Profa. Dra. Suraya Gomes Novais-Shimano - UFTM/MG

Profa. Dra. Suzel Regina Ribeiro Chavaglia - UFTM/MG

Profa. Dra. Sybelle de Sousa Castro - UFTM/MG

Profa. Dra. Taka Oguisso - USP/SP

Profa. Dra. Teresinha Covas Lisboa - Faculdade Araguaia/GO

Profa. Dra. Zenith Rosa Silvino - UFF/RJ

\section{DIRETORIA EXECUTIVA}

Profa. Dra. Jurema Ribeiro Luiz Gonçalves - UFTM/MG

Profa. Dra. Lislei Jorge Patrizzi - UFTM/MG

Profa. Ms. Emiliane Silva Santiago - UFTM/MG

Profa. Ms. Marina Leandrini - UFTM/MG

Profa. Ms. Paula Tatiana Cardoso - UFTM/MG

Prof. Mts. Rodrigo Eurípedes da Silveira - UFTM/MG

Esp/Mts. Alexandre Marcelo Costa de Oliveira - UFTM/MG

Esp/Mts. Carla Maria Silvano - UFTM/MG

Esp/Mts. Dayane Aparecida Viana - UFTM/MG

Esp/Mts. Francielle Toniolo Nicodemos da Silva - UFTM/MG

Esp/Mts. Michelle Helena Pereira de Paiva - UFTM/MG

Esp/Mts. Pollyana Pagliario - UFTM/MG

Mts. Talita Inácio Martins - UFTM/MG

\section{BIBLIOTECÁRIA}

Sônia Maria Rezende Paolinelli (CRB-6/1191) - UFTM/MG

\section{ASSESSORIA DE IMPRENSA}

João Pedro Vicente (MTb 11885/MG) - UFTM/MG

ASSESSORIA DE REVISÃO E DIAGRAMAÇÃO

Dalva Pereira Silva - UFTM/MG

Lúcia Helena Coimbra Amaral - UFTM/MG 


\section{Sumário}

Editorial

A importância de uma revista científica na interface de Saúde e Ciências Sociais e Humanas.

Álvaro da Silva Santos

Modelo RE-AIM: Tradução e Adaptação cultural para o Brasil.

RE-AIM framework: Translation and Cultural adaptation to Brazil

Modelo RE-AIM: Traducción y Adaptación Cultural para el Brasil

Fabio Araujo Almeida, Fabiana de Almeida Brito, Paul Andrew Estabrooks

Estado nutricional de escolares de uma escola pública estadual.

Nutritional status of schoolchildren from a state public in Uberaba, Minas Gerais, Brazil

Estado nutricional de los estudiantes de una escuela pública estatal em Uberaba, Minas Gerais, Brasil

Juliana Bucci Silva Matos de Brito, Isabel Aparecida Porcatti de Walsh, Renata Damião

O morrer para graduandos em Enfermagem: a contribuição da Psicologia

The die for undergraduates in nursing: the contribution of psychology

El morir para los estudiantes de enfermería: la contribución de la psicología

Gheysa Alves Mendonça, Valtuir Duarte Souza Júnior, Nivea Lourenço Santos Correio, Álvaro Silva Santos

Diagnósticos de enfermagem frequentes em idosos residentes na área de abrangência de uma Estratégia Saúde da Família.

Nursing diagnoses frequently in elderly residents in an the area of coverage of family health strategy Diagnósticos de enfermería frecuentes en los residentes mayores en una area de cobertura de una estrategia de salud familiar

Gerson de Souza Santos, Marina Borges Teixeira

A deficiência visual e o processo de construção da cidadania: um estudo no Instituto de Cegos do Brasil Central de Uberaba.....

The visual impairment and the process of construction of citizenship: a study in the Institute for the Blind of Brasil Central of Uberaba, Minas Gerais, Brazil

La debilitación visual y el proceso de construcción de la ciudadanía: un estudio en el Instituto para Ciegos del Brasil Central de Uberaba, Minas Gerais, Brasil

Claudia Helena Julião, Marlene Aparecida Manzan Paiva, Suraya Gomes Novais Shimano, Verena Conti, Zilda Cristina dos Santos

Internações e óbitos de idosos por influenza no estado de Minas Gerais...............................50 Hospitalizations and deaths among the elderly from influenza in the state of Minas Gerais, Brazil Hospitalizaciones y muertes entre los ancianos por influenza en el estado de Minas Gerais, Brasil

Dayane Aparecida Viana, Lilane Maria Alves Silva, Leiner Resende Rodrigues

Desenvolvimento da linguagem em crianças surdas: uma revisão integrativa. .56 Language development in deaf children's: an integrative review

Desarrollo del lenguaje en niños sordos: una revisión integradora

Nathália Fernandes Minaré, Fabio Scorsolini-Comin, Manoel Antônio dos Santos 